\title{
A Second Order Problem with Nonlocal Boundary Conditions*
}

\author{
Todor D. Todorov
}

\begin{abstract}
A second order elliptic problem with nonlocal boundary conditions is the object of interest in this paper. The strong problem is reduced into a unconstrained minimization one. Sufficient conditions for existence and uniqueness of the weak solution are proved. A priory estimates for the weak solution are obtained.
\end{abstract}

Keywords: well-posedness, unconstrained minimization problem, nonlocal boundary conditions.

\section{Introduction and setting of the problem}

Plenty of problems with nonlocal boundary conditions have been studied $[2,3,4,5]$ because of their various applications in the engineering sciences. A more complicated nonlinear $p$ Lapalcian problem with nonlocal boundary conditions has been studied by Amster and De Napoli [1].

This paper is devoted to a specific second order problem with nonlocal boundary conditions, which is not studied for well-posedness up to now. Let us start with some basic definitions and denotations. The domain $\Omega$ is an open polygonal subset of $\mathbf{R}^{2}$. The real Sobolev space $H^{k}(\Omega)$ is provided with the norm $\|\cdot\|_{k, \Omega}$ and the seminorm $|\cdot|_{k, \Omega}$. We denote the $k$-th Fréchet derivative of a map $F(x)$ by $D^{k} F(x)$ and the $L^{2}(\Gamma)$-scalar product by

$$
<u, v>=\int_{\Gamma} u v d l
$$

We define the space $\mathbf{V}=H_{0}^{1}(\Omega)$ and the second-order elliptic linear operator

$$
L u=-\sum_{i, j=1}^{2} \frac{\partial}{\partial x_{j}}\left(a_{i j} \frac{\partial u}{\partial x_{i}}\right)+a \cdot u, \operatorname{dom} L=C_{0}^{2}(\bar{\Omega}) .
$$

Manuscript received May 12, 2018; accepted September 15, 2018.

Todor D. Todorov is with the Department of Mathematics and Informatics, Technical University, 5300 Gabrovo, Bulgaria, e-mail: t.todorov@yahoo.com

*This work is supported by Technical University of Gabrovo, research grant 1814C/2018. 
The coefficients $a_{i j}(x)$ and $a(x)$ belong to $C^{1}(\bar{\Omega}), a_{i j}=a_{j i}, i, j=1,2$ and $a(x) \geq a_{0}>$ $0, \forall x \in \Omega$. We assume that $L$ is uniformly elliptic, i.e. there exists a constant $\alpha>0$ such that

$$
\alpha \sum_{i=1}^{n} \xi_{i}^{2} \leq \sum_{i, j=1}^{2} a_{i j}(x) \xi_{i} \xi_{j}, \quad \forall \xi, x \in \mathbf{R}^{2}
$$

The set $\Gamma_{0}$ is obtained from the boundary $\Gamma=\partial \Omega$ by removing the vertex points where the normal derivative does not make sense. Let $v_{i}$ be the $i$-th component of the outward unit normal on $\Gamma_{0}$. The problem of interest is

$$
\mathscr{S}:\left\{\begin{array}{c}
\text { Find } u \in C_{0}^{2}(\bar{\Omega}) \text { satisfying : } \\
L u=f \text { in } \Omega, \\
\frac{\partial u}{\partial v}=<u, u>u \text { on } \Gamma_{0}
\end{array},\right.
$$

where the right hand side $f$ satisfies:

$$
f \in L^{2}(\Omega),\|f\|_{0, \Omega} \neq 0
$$

and

$$
\frac{\partial u}{\partial v}=\sum_{i, j=1}^{n} a_{i j} \frac{\partial u}{\partial x_{j}} v_{i}
$$

stands for the conormal derivative.

\section{The weak formulation}

The weak form of the problem $(\mathscr{S})$ is related with the application of the finite element method for determine of approximate solutions of $\mathscr{S}$. Multiplying the equation $L u=f$ by $v \in \mathbf{V}$ and applying Green's theorem we obtain

$$
\mathscr{W}:\left\{\begin{array}{c}
\text { Find } u \in \mathbf{V} \text { such that } \\
a(u, v)+<u, u><u, v>=(f, v) \quad \forall v \in \mathbf{V} \quad \text { in } \Omega,
\end{array}\right.
$$

where $a(u, v)$ is the bilinear form

$$
a(u, v)=\int_{\Omega} \sum_{i, j=1}^{2} a_{i j}(x) \frac{\partial u}{\partial x_{i}} \frac{\partial v}{\partial x_{j}} d x+\int_{\Omega} a(x) u v d x,
$$

and $(\cdot, \cdot)$ is the $L^{2}(\Omega)$-scalar product. Since $L$ is a linear continuous and uniformly $\mathbf{V}$-elliptic operator there exist positive constants $\underline{\alpha}$ and $\bar{\alpha}$ such that

$$
\underline{\alpha}\|u\|_{1, \Omega}^{2} \leq a(u, u), \quad a(u, v) \leq \bar{\alpha}\|u\|_{1, \Omega}\|v\|_{1, \Omega}, \forall u, v \in \mathbf{V} .
$$


The objective functional

$$
J(v)=\frac{a(v, v)}{2}+\frac{<v, v>^{2}}{4}-F(v)
$$

with

$$
F(v)=(f, v)
$$

is used in order to compile the following unconstrained minimization problem

$$
\mathscr{M}: \underset{v \in \mathbf{V}}{\operatorname{argmin}} J(v)
$$

The problem $\mathscr{M}$ is associated with the weak formulation $(\mathscr{W})$ since

$$
D J(u) v=a(u, v)+<u, u><u, v>-(f, v)
$$

Theorem 2.1. If the linear operator $L$ is uniformly $\mathbf{V}$-elliptic and $f$ satisfies the condition (2) then the problem $(\mathscr{M})$ has a unique solution.

Proof. In order to prove the theorem we have to show that the functional $J(v)$ is bounded below, coercive and convex. From (3) and the Cauchy inequality we conclude that

$$
J(v) \geq \frac{\alpha}{2}\|v\|_{1, \Omega}^{2}-\|f\|_{0, \Omega}\|v\|_{1, \Omega}
$$

Therefore, $J(v)$ is bounded below and coercive. The second Fréchet derivative

$$
D^{2} J(u)(v, w)=a(v, w)+2<u, v><u, w>+<u, u><v, w>
$$

is nonnegative if $v=w$, which implies the convexity of $J(v)$.

Corollary 1. If the conditions of Theorem 2.1 hold then the problem (W) has a unique solution.

Proof. The assertion of the corollary follows from the fact that the problem $(\mathscr{W})$ is equivalent to $D J(u) v=0 . \square$

The next lemma describes validity of a variational inequality applicable in error analysis of approximate solutions.

Lemma 1. If $L$ is uniformly $\mathbf{V}$-elliptic and the condition (2) hold then

$$
a(u, v-u)+<u, u><u, v-u>\geq F(v-u), \forall v \in \mathbf{V},
$$

where $u$ is the unique stationary point of the functional $J(u)$. 
Proof. Since $u$ is the unique solution of $(\mathscr{M})$ the inequality

$$
J(u+t w)-J(u) \geq 0, \forall w \in \mathbf{V}
$$

implies

$$
D J(u) w \geq 0 .
$$

Replacing $w=v-u$ in (6) and applying (4) we obtain (5)

Theorem 2.2. If the conditions of Theorem 2.1 hold then

$$
\|u\|_{1, \Omega} \leq C\|f\|_{0, \Omega}
$$

Proof. The inequality (7) follows from (5) with $v=0$.

\section{Conclusion}

A linear second order problem with a nonlocal boundary conditions is studied for wellposedness in this paper. A weak form of the problem of interest is obtained. The weak formulation of the initial problem is transformed into unconstrained minimization problem. Convexity of the objective functional is proved. Sufficient conditions for existence and uniqueness of the weak solution are derived. A variational inequality concerning the weak solution is proved.

\section{References}

[1] P. Amster and P. De Napoli, A nonlinear second order problem with a nonlocal boundary condition, Abstract and Applied Analysis, Volume 2006 (2006), Article ID 38532, pp. 1-11.

[2] F. Kanca, Inverse coefficient problem for a second-order elliptic equation with nonlocal boundary conditions, Mathematical Methods in the Applied Sciences, Volume 39, Issue 11, 2016, pp. 3152-3158.

[3] C. Nie, S. Shu, H. Yu, Q. An, A high order composite scheme for the second order elliptic problem with nonlocal boundary and its fast algorithm, Applied Mathematics and Computation, Volume 227, 2014, pp. 212-221.

[4] C. Nie, H. Yu, Some error estimates on the finite element approximation for two-dimensional elliptic problem with nonlocal boundary, Applied Numerical Mathematics, Vol. 68, 2013, pp. 31-38.

[5] H. De Schepper, M. Slodivcka, Recovery of the boundary data for a linear second order elliptic problem with a nonlocal boundary condition, ANZIAM Journal, vol. 42, 2000, pp. 518-535. 